Draft Version SePtember 23, 2018

Preprint typeset using $\mathrm{LAT}_{\mathrm{E} X} \mathrm{X}$ style emulateapj v. 12/16/11

\title{
NEW ORBITS BASED ON SPECKLE INTERFEROMETRY AT SOAR. II. ${ }^{1}$
}

\author{
ANDREI TOKOVININ \\ Cerro Tololo Inter-American Observatory, Casilla 603, La Serena, Chile \\ Draft version September 23, 2018
}

\begin{abstract}
Orbits of 44 close and fast visual binaries are computed using the latest speckle observations; 23 orbits are determined for the first time, the rest are revisions, some quite substantial. Six combined orbits use radial velocities. The median period is 15.6 years, the shortest period is one year. Most stars are nearby late-type dwarfs. Dynamical parallaxes and estimates of the masses are derived from the orbital elements and the photometry of the components.

Keywords:
\end{abstract}

\section{INTRODUCTION}

This paper presents new or updated orbits of 44 binary systems or subsystems. It is based on speckle interferometric measurements made at the $4.1 \mathrm{~m}$ Southern Astrophyisical Research (SOAR) telescope (Tokovinin et al. 2010a,b; Tokovinin 2012; Tokovinin et al. 2014, 2015, 2016) combined with archival data collected in the Washington Double Star Catalog, WDS (Mason et al. 2001). It continues previous work on binary orbits resulting from the SOAR speckle program and follows the template of the Paper I (Tokovinin 2016), where the motivation is discussed. Briefly, calculation of binary orbits is part of the astronomical infrastructure and visual orbital elements are used in many areas. The state of the art is reflected in the Sixth Catalog of Visual Binary Orbits, VB6 (Hartkopf, Mason \& Worley 2001). ${ }^{2}$

Accurate parallaxes from Gaia (Lindegren et al. 2016) are expected to yield good mass measurements of visual binaries with reliable orbits. However, the short 5 year duration of the Gaia mission will make it difficult to disentangle orbital and parallactic motions of the photo-center and might lead to biased parallaxes of visual binaries, as happened with the Hipparcos mission (Shatskii \& Tokovinin 1998; Söderhielm 1999). Therefore, orbital elements based on ground-based data will be essential for getting correct astrometry of binary stars from Gaia. The Gaia DR1 explicitly avoids binaries and gives parallaxes only for a few stars featured in this paper.

\section{ORBITAL ELEMENTS}

The methods are identical to those of Paper I, so they are outlined only briefly. Orbital elements and their errors are determined by least-squares fitting using the IDL code ORBIT. ${ }^{3}$ When no prior orbit is available, the initial approximation is chosen interactively, considering the observed motion of each pair. This step is largely heuristic. Sometimes the same data can be represented by several very different orbits. In those cases, additional information such as the mass sum, radial velocities (RVs), times

\footnotetext{
Electronic address: atokovinin@ctio.noao.edu

${ }^{1}$ Based on observations obtained at the Southern Astrophysical Research (SOAR) telescope.

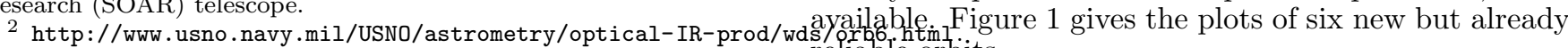

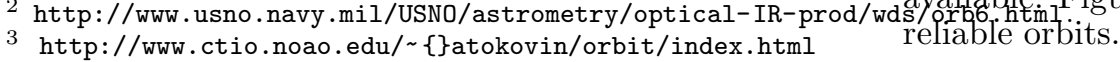
brackets. The last column contains references to the previously computed visual and spectroscopic orbits, when
}

of non-resolutions, etc. is used to select the correct orbit; several examples are found in Section 3 .

The second step is the least-squares refinement (fitting) of the elements. The weights are inversely proportional to the square of the measurement errors, which are assigned subjectively based on the technique used. Some outliers are given very low weight, otherwise they would distort the orbit. For pairs observed at SOAR, the typical position errors are 2 mas in the $y$ filter and 5 mas in the $I$ filter. They are larger for difficult binaries which are either close to the diffraction limit or very faint. When the orbit is not well constrained by the data, some orbital element, e.g. eccentricity or inclination, is fixed to the value that results in the expected mass sum, given the HIP2 parallax (van Leeuwen 2007). The choice of the element to fix is individual, depending on the coverage and the orbit configuration. Its value is selected by trial and error while fitting the remaining elements and checking the mass sum. Considering potential parallax biases mentioned above and the uncertainty of the estimated masses, this approach is hazardous; it is used only for preliminary low-quality orbits. RVs, when available from the literature, are used jointly with the positional measurements, improving substantially the quality of such combined orbits.

Table 1 lists the orbital elements and their errors in common notation $\left(P-\right.$ orbital period, $T_{0}$ - epoch of periastron in Besselian years, $e$ - eccentricity, $a$ - semimajor axis, $\Omega$ - position angle of the node for the equinox J2000.0, $\omega$ - argument of periastron, $i$-inclination). The first column gives the WDS code of the binary and, in the following line, its Hipparcos number. The system identifier adopted in the WDS ("discoverer code") is given in the second column. For each pair, the first line contains the orbital elements, while the following line gives their formal errors. The orbit grades are given in the VB6 system, where grades 4 and 5 mean preliminary orbits and grade 1 are definitive and accurate orbits. Those grades were kindly computed by the Referee. However, this grading system does not account for the RV data that strengthen combined visual-spectroscopic orbits. Alternative unofficial grades are provided for such orbits in 

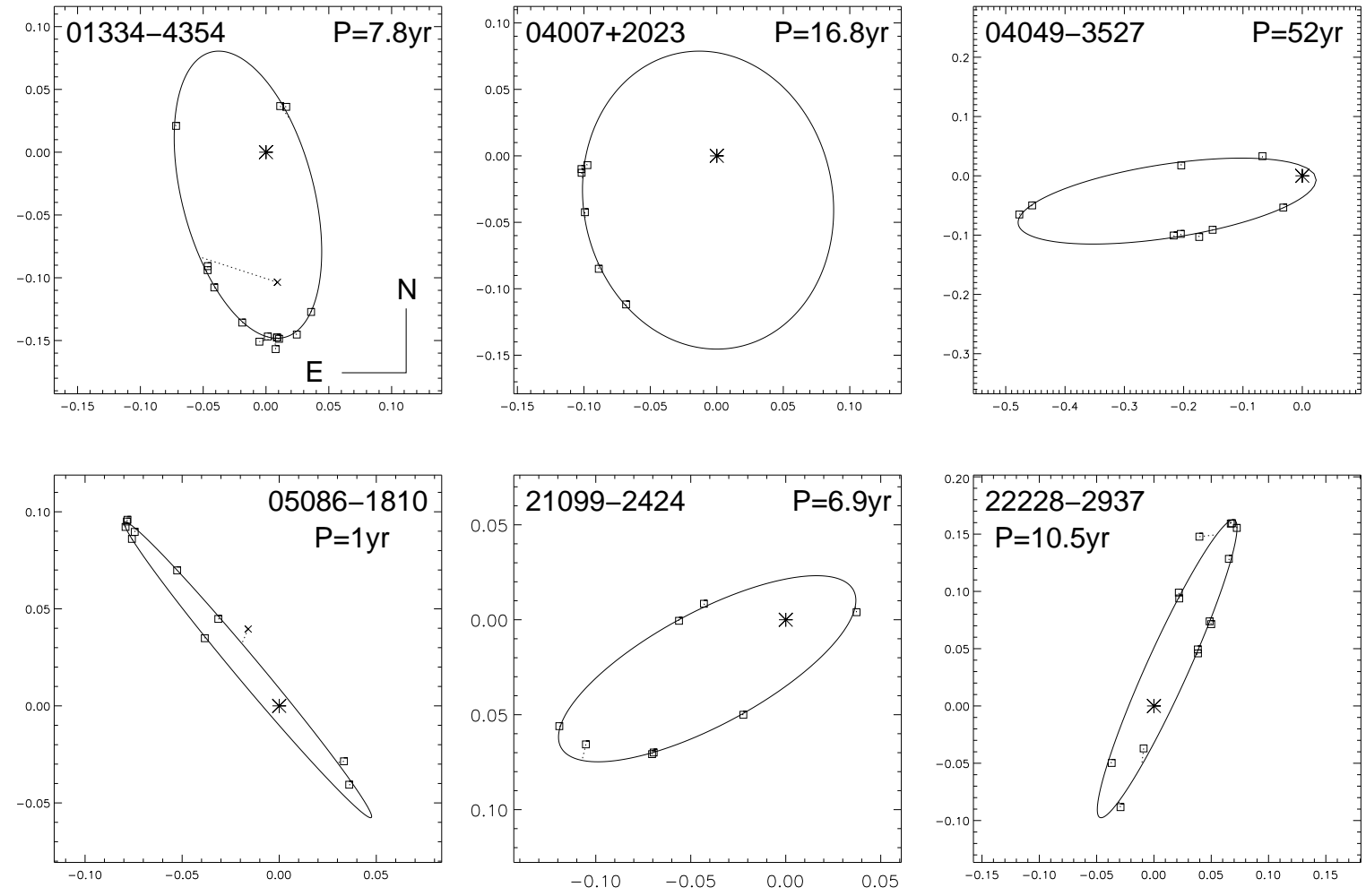

Figure 1. New reliable orbits of six binaries. In each plot, the primary component (star) is at the coordinate origin, with the scale in arcseconds. The ellipse depicts the orbit, the squares connected to the ellipse are the measurements. Measurements with large deviations and low weight are plotted as crosses.

Individual observations and residuals are listed in Table 2, available in full electronically. It contains still unpublished measures made at SOAR in 2016 and 2017, while some published SOAR measures were reprocessed. Its first column identifies the binary by its WDS code (for multiple systems, the discovery codes and component designations are found in Table 1). Then follow the time of observation $T$ in Besselian years, the position angle $\theta$ for the time of observation (correction for precession is done internally during orbit calculation), the separation $\rho$ in arcseconds, and the measurement error $\sigma$. Unrealistically large errors are assigned to the discarded observations to give them zero weight, but those data are still kept in the Table. The following two columns of Table 2 contain the residuals $\mathrm{O}-\mathrm{C}$ in angle and separation. The last column contains flags specifying the source of the data: $\mathrm{M}$ for historic micrometer measures, $\mathrm{H}$ for the Hipparcos measure, $\mathrm{S}$ for speckle interferometry at SOAR, I for speckle interferometry from other sources found in the online Fourth Catalog of Interferometric Measurements of Binary Stars (Hartkopf et al. 2001). ${ }^{4}$

Table 3 provides additional information, namely the the spectral type as given in Hipparcos or SIMBAD and the HIP2 parallax $\pi_{\mathrm{HIP} 2}$, to be compared to the dynamical parallax $\pi_{\text {dyn }}$ in the next column. The latter is evaluated by the Baize-Romani method, as explained in Paper I. The components' masses $\mathcal{M}_{1}$ and $\mathcal{M}_{2}$ obtained in this procedure are listed in the columns (6) and (7) of Table 3. Asterisks mark the dynamical parallaxes derived from reliable orbits of grade 3 or better.
The combined $V$ magnitude and $V-I_{C}$ color index in columns (8) and (9) are taken mostly from the Hipparcos catalog. For HIP $32366 \mathrm{~B}$, the combined magnitudes refer to the secondary subsystem, while $V-I_{C}=2.7 \mathrm{mag}$ is assumed to match the estimated spectral type. The last four columns of Table 3 provide differential photometry resulting from the SOAR speckle interferometry, where the filters $y$ and $I$ have the central wavelengths and the bandwidths of $543 / 22$ and $788 / 132 \mathrm{~nm}$, respectively. The $\Delta y$ and $\Delta I$ are average values, while $\sigma_{\Delta y}$ and $\sigma_{\Delta I}$ stand for the rms scatter of magnitude difference in each filter if measured several times, indicating the internal consistency of the differential photometry. For some pairs this scatter is as good as $0.1 \mathrm{mag}$, but it can be much larger. The speckle differential photometry is unreliable for very close pairs at or below the diffraction limit. For faint or wide binaries, $\Delta m$ can be systematically overestimated. Despite these caveats, speckle interferometry at SOAR is the only source of differential photometry for some close binaries.

When both $\Delta y$ and $\Delta I$ are measured, the crude empirical linear relation $\Delta I \approx 0.7 \Delta y$ holds for dwarf stars observed at SOAR, allowing to estimate differential magnitudes in both filters even when measurements in only one filter are available. It is also reasonable to assume that $\Delta V \approx \Delta y$ and $\Delta I_{C} \approx \Delta I$. Therefore, magnitudes and colors of each binary component can be estimated using combined and differential photometry.

Figure 2 shows the $\left(M_{V}, V-I_{C}\right)$ color-magnitude diagram $(\mathrm{CMD})$ where only binaries with $\pi_{\mathrm{HIP} 2}>4$ mas are plotted. For reference, the $1 \mathrm{Gyr}$ isochrones from Dotter et al. (2008) and Baraffe et al. (2015) are shown.

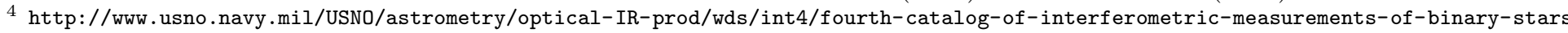




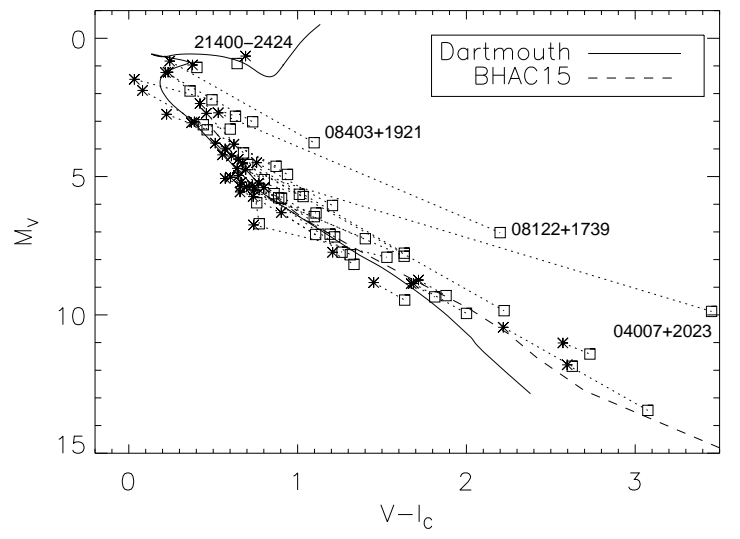

Figure 2. Color-magnitude diagram. The primary and secondary components of each pair are plotted as asterisks and squares, respectively, and connected by dotted lines. The full line is the Dartmouth isochrone (Dotter et al. 2008), the dashed line is the BHAC15 (Baraffe et al. 2015) isochrone, both for 1 Gyr age and solar metallicity. Four secondary components located above the main sequence are labeled by their WDS codes.

\section{NOTES ON INDIVIDUAL BINARIES}

Brief comments on all binaries are provided in this Section.

00135-3650. This is a nearby pair of M0.5V dwarfs. With only four measures, the orbit is obviously preliminary, although this system has made two revolution since its discovery by Hipparcos.

00164-7024. This first preliminary orbit lacks coverage.

00258+1025. The pair of G-type dwarfs HDS 57 approaches the periastron, so fast motion is expected in the coming years.

01334-4354. This is the first, but well-defined orbit with $P=7.77$ years (Figure 1). The SOAR data sample one full period. The Hipparcos measure is off because of the short period. The primary mass of $1 \mathcal{M}_{\odot}$ is too large for the spectral type K0.5V listed in SIMBAD. Both components are located on the main sequence in the CMD.

02166-5026. The pair TOK 185, discovered at SOAR in 2011, has covered more than a half of its 14 year orbit, including the periastron; the orbit is updated.

02390 - 5811. This very fast $(P=6$ years $)$ pair of M0V dwarfs was near the periastron in 2016.9 and has made four revolutions since its discovery by Hipparcos.

$04007+2023$. This star is a member of the Hyades cluster and a single-lined spectroscopic binary with a period of 16.7 years (Griffin 2012). The SOAR measures cover only a part of the ellipse, but the combined orbit using the Griffin's RVs is well defined (Figure 1). Griffin remarked on the potential resolution of this pair: "The difference in luminosity in the $V$ band may be expected to be around five magnitudes - an impossible prospect visually..." In fact the binary was resolved at SOAR a year after the publication of the Griffin's paper. Indeed, $\Delta y=5.2 \mathrm{mag}$, but the pair is far from being "impossible". The secondary component is located well above the main sequence in the CMD in Figure2, having $V-I_{C}=3.45 \mathrm{mag}$. However, the five measurements of $\Delta I$ have a large scatter of $0.73 \mathrm{mag}$, so the unusually red color of the secondary component needs confirmation. The RV amplitude corresponds to the secondary mass of $0.58 \mathcal{M}_{\odot}$ if the primary mass is $1.14 \mathcal{M}_{\odot}$, in agreement with the masses in Table 3 .

04049-352\%. This is a visual triple system where the outer pair A,BC is the $1^{\prime \prime}$ binary I 152 with the estimated period of $\sim 300$ years. Although the 52 year orbit of the subsystem B,C is computed for the first time, it is well constrained (Figure 11); this pair, discovered in 1990.9 at 0 ". 48 , passed through the periastron in 2009.7 and now opens up again. Its large eccentricity of 0.92 could be caused by the outer system.

04074-6413. The spectroscopic elements from Jenkins et al. (2015) are fixed and only the period and the "visual" elements are fitted. Unfortunately, no RVs are given in that paper for computing the combined orbit. The spectroscopic mass of the secondary component, $0.53 \mathcal{M}_{\odot}$, matches the mass in Table 3 .

04107-0452. This is HIP 19508, HD 26441, or ADS 3041. The previous 20 year visual orbit with high eccentricity is that of Tokovinin et al. (2014). A concordant double-lined spectroscopic orbit based on 36 years of RV coverage has been published by Griffin (2015) in a paper which is, unfortunately, ignored by SIMBAD. The orbit here is a combined one based on the Griffin's RVs and the speckle data, with the visual micrometer measures given a very low weight. The combined orbit is highly accurate and deserves the grade 1 ; its spectroscopic elements $\left(K_{1}=11.61, K_{2}=12.55, V_{0}=26.59\right.$ $\mathrm{km} \mathrm{s}^{-1}$ ) are close to those given by Griffin. The combined orbit leads to the masses of $1.120 \pm 0.017$ and $1.036 \pm 0.016$ $\mathcal{M}_{\odot}$ for the primary and secondary components, respectively. The orbital parallax of $17.22 \pm 0.31$ mas is more accurate than the HIP2 parallax $16.09 \pm 0.65$ mas. The stars are slightly evolved off the main sequence, hence the masses in Table 3 are somewhat over-estimated. After submission of this paper we became aware of the work by Docobo et al. (2017) who proposed a similar visual orbit. However, they have not computed the combined orbit and apparently used a different weighting scheme. Their orbit is consequently less accurate. While the combined orbit gives $a=0^{\prime \prime} 167 \pm 0.002$ and $i=69^{\circ} .0 \pm 0.5$, the orbit by Docobo et al. gives $a=00^{\prime \prime} 161 \pm 0.002$ and $i=66.4 \pm 0.5$; the difference exceeds the formal errors.

$04422+0259$. The updated 58 year orbit of A 2424 is now well defined by the speckle data alone; only the first visual measure is used to constrain the period.

05086-1810. The first orbit of WSI 72 with a remarkably short (for a resolved binary) period of one year (Figure 1) is well defined by the 11 speckle measures, leaving the rms residuals of only 1.4 mas (the latest 2016.96 measure at the diffraction limit deviates more, so it was given a low weight). The dynamical parallax of 118.5 mas appears more accurate than the HIP2 parallax of 108 mas, obviously biased by the photocenter motion. The deduced masses of $0.3 \mathcal{M}_{\odot}$ are normal for the M5V dwarfs.

05103-0736. The nearly circular 37.6 year orbit of HIP 24076 by Hartkopf et al. (2012) fits the measures well, although it predicts a too small mass sum of $1.2 \mathcal{M}_{\odot}$. Double lines were noted by Nordström et al. (2004). Spectroscopic monitoring by N. Gorynya (2017, private communication) did not show any substantial velocity variability during four years, meaning that the system is not triple. Therefore, the orbit of the visual binary is likely eccentric, with half the period. The new 18.9 year orbit with $e=0.82$ fits the interferometric data 
very well (rms residuals 2 mas). It yields the mass sum of $2.7 \mathcal{M}_{\odot}$, appropriate for a couple of F8V stars. Observations around the next periastron in 2018.6 are expected to confirm the eccentric orbit and to refute the circular one. The RV measurements near the periastron can yield accurate masses.

05525-0217. The 12 year orbit of HDS 787, updated from (Tokovinin et al. 2014), is now definitive, with a good coverage. Four speckle measures made at the $3.5 \mathrm{~m}$ WYIN telescope in 1999-2004 were assigned low weights, although the reason why they are discrepant is not clear. The Hipparcos magnitude difference $\Delta H p=0.87 \mathrm{mag}$ is underestimated (in fact $\Delta y=1.6 \mathrm{mag}$ ), which is normal for such a close (97 mas) pair, well below the diffraction limit of the $30 \mathrm{~cm} \mathrm{Hipparcos}$ aperture.

06454-3148. This is a nearby triple system GJ 245.1 where the faint pair of low-mass dwarfs Ba,Bb at 1".4 from the main star HIP 32366 has been discovered in 2005 by Ehrenreich et al. (2010). Apart from the discovery paper, the only published observations are those made at SOAR. The magnitude difference between the F7V primary and the late- $\mathrm{M}$ component $\mathrm{Ba}$ is $\Delta I=$ $6.57 \pm 0.47 \mathrm{mag}$, meaning that the faint pair $\mathrm{Ba}, \mathrm{Bb}$ is just above the detection limit at SOAR. The 6.9 year orbit of $\mathrm{Ba}, \mathrm{Bb}$ is slightly updated with respect to (Tokovinin et al. 2015). With the Gaia parallax of 39.61 mas it corresponds to the mass sum of $0.54 \mathcal{M}_{\odot}$. The mass of $\mathrm{Ba}$ is therefore close to $0.30 \mathcal{M}_{\odot}$ and implies an M3.5V dwarf. Its magnitude $I=11.9$ mag according to our photometry roughly matches the standard relations and the distance modulus of $2.0 \mathrm{mag}$. Given the lack of the $V$-band photometry, Table 3 adopts the combined magnitude and color appropriate for the masses, making the dynamical parallax meaningless.

06533-1902. The first 36 year orbit of CHR 169 is reasonably well constrained, despite the large gap in its coverage between 1996 and 2014. The pair is actually going through the periastron.

07269+2015. The orbit of CHR 26 by Olevic \& Jovanovic (1998) with $P=14.17$ years did not match the latest measures; it is revised to $P=8.6$ years.

08122+1739. This is $\zeta$ Cnc C, a member of the hierarchical multiple system. The 59 year visual orbit of A,B and the crude visual orbit of AB,C (period 1115 years) have been computed previously. Here the preliminary visual orbit of $\mathrm{Ca}, \mathrm{Cb}$ by Riddle et al. (2015) is replaced by the combined 17 year orbit using the RVs from Griffin (2000). Our orbit agrees roughly with the astrometric orbit computed by Heintz (1996). However, only a small sector of the visual orbit is covered by accurate measures, whereas the remaining measures have large errors and fit the orbit poorly. The HIP2 parallax of 39.9 mas leads to the large mass sum of $2.45 \mathcal{M}_{\odot}$ and supports the conclusion of Hutchings et al. (2000) that the masses of $\mathrm{Ca}$ and $\mathrm{Cb}$ are comparable, with $\mathrm{Cb}$ being a close pair of M2 dwarfs. The unusually red color of $\mathrm{Cb}$ ( $\Delta y=4.3, \Delta I=2.7, \Delta K=0.2 \mathrm{mag}$, see Figure 2) is explained by its binarity. To reconcile the RV amplitude with the mass sum, we fixed the inclination to $150^{\circ}$. The RV amplitude and the inclination in the combined orbit correspond to the $\mathrm{Cb}$ mass of $1.25 \mathcal{M}_{\odot}$ if the mass of $\mathrm{Ca}$ is $1.2 \mathcal{M}_{\odot}$. The dynamical masses in Table 3 do not account for the binarity of $\mathrm{Cb}$ and are therefore incor- rect. Further speckle monitoring of this interesting pair is obviously needed.

$08403+1921$. This is an A9V triple system in the Praesepe cluster (NGC 2635), where the visual secondary $\mathrm{B}$ is a double-lined spectroscopic binary with a period of 48.7 days. The outer orbit by Olevid (2002) with $P=13.2$ years corresponds to the very large mass sum of $54 \mathcal{M}_{\odot}$, using the Gaia parallax of 4.71 mas; it does not match the latest measures. The available data can be represented by a very eccentric $(e=0.84)$ orbit with $P=10.6$ years or by a nearly circular orbit with $P=21.6$ years, both with the inclination of $90^{\circ}$. However, neither of those orbits match the separation of 115 mas measured in 1982.25 by lunar occultations. Therefore we prefer the third orbit with a longer period of 35.5 years, compatible with the occultation measure, although it fits the speckle data slightly worse than the two shorter-period orbits. The mass sum in the 35 year orbit is reduced to $21 \mathcal{M}_{\odot}$ with the Gaia parallax or to $6 \mathcal{M}_{\odot}$ with the HIP2 parallax of 7.15 mas. The HIP2 parallax places the primary component on the main sequence turnoff (Figure 2). The problem with this binary is the lack of speckle monitoring between 1997 and 2016; during this period, only one measure was made.

Abt \& Willmarth (1999) measured the RVs of the primary broad-lined component which show a positive trend during the two year time span of their data. The trend is compatible with all visual orbits and does not help to choose between them. The RVs of the center of mass of the secondary computed from their 48 day spectroscopic orbit have a large scatter and throw some doubt on that orbit, which the authors themselves call tentative.

08447-4238. This is a very well covered orbit with $P=2.26$ years. The HIP2 parallax is likely biased by the short period.

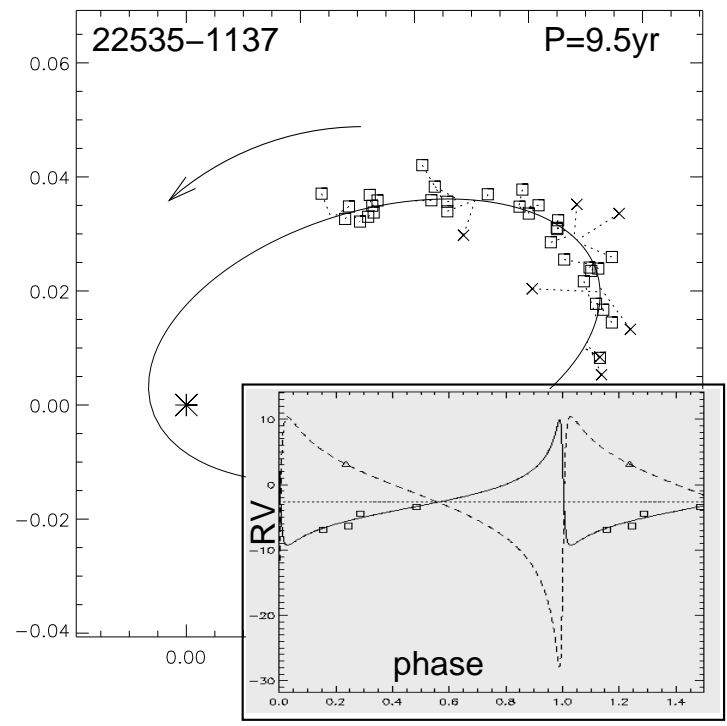

Figure 3. The eccentric orbit of HR 8704 with $P=9.5$ years. The insert shows the RV curve.

09252-1258. The 27 year orbit of WSI 73 by Tokovinin et al. (2015) is radically revised here to $P=13$ years after this pair of $\mathrm{K} 1 \mathrm{~V}$ dwarfs became unresolved at SOAR in 2015 and opened up in 2016. It will close again in 2019. The new orbit appears secure. 
10121-0241. DEL 3 is a pair of nearby M0V dwarfs for which we propose the first, still preliminary edge-on orbit with a short 6.4 year period. The eccentricity is fixed to tune the mass sum.

10294+1211. The orbit by Cvetkovic et al. (2016) $(P=23.36$ year $)$ strongly disagrees with the data and is radically revised to $P=15.6$ years. Although the pair has made nearly two revolutions since its discovery in 1991, the coverage remains scarce.

10529-1717. The updated 15 year orbit of HDS 1556 is now definitive, with both extremities covered. The pair is closing down and will pass through the periastron in 2018.55 .

11420-1701. This visual triple system was discovered at SOAR in 2013. The first preliminary 21 year orbit of the inner pair TOK 384 is proposed here (obviously, it still lacks coverage), while the estimated period of the outer 1 .' 1 companion is about 300 years.

12485-1543. The orbit of the 2.6 year inner pair $\mathrm{Aa}, \mathrm{Ab}$, first computed by Tokovinin et al. (2014), is updated using new speckle measures and the unpublished RVs of both components measured by D. Latham (2012, private communication). The outer component in this nearby triple system, at 2.'6 separation, has an estimated period of 400 years. Horch et al. (2017) published recently a similar visual orbit, apparently unaware of the paper by Tokovinin et al. (2014). Compared to Horch et al., the coverage is extended here by two years and the RVs are added, making the new orbit more accurate. The combined orbit yields the masses of $0.96 \pm 0.08$ and $0.75 \pm 0.07 \mathcal{M}_{\odot}$ and the orbital parallax of $36.7 \pm 1.1$ mas.

13137-6248. The 25.6 year period of HDS 1852 is well defined because it is now passing through the same part of the orbit where it was discovered in 1991. Other orbital elements are still preliminary.

14330-4224. The pair of A7V stars HDS 2054 passed through the periastron around 1998 without being observed and is now slowly opening. Its 66 year orbit is preliminary, with a fixed eccentricity. The mass sum of $3.3 \mathcal{M}_{\odot}$ is less than expected from the spectral type. If the Hipparcos measure is flipped, the observed motion looks almost like a straight line, although its curvature is still significant. A rough circular orbit with $P=222$ yr and $a=0$ ". 33 can be fitted to this arc.

15245-1322. The preliminary 82 year orbit of $\mathrm{K} 7 \mathrm{~V}$ dwarfs appears reasonably well constrained by the measures at SOAR and by those of Horch et al. (2017).

15339-1700. The first 60 year orbit of HDS 2185 by Tokovinin (2012) is updated here, as half of it is now covered.

17176+1025. The orbit by Cvetkovic (2013) is revised to $P=46$ years. This chromospherically active star V2369 Oph is possibly young; it contains an eclipsing subsystem with a period of 0.655 .

17195-5004. The measures of FIN 356 with nearly equal components, allowing arbitrary quadrant changes, can be fitted by several different orbits. However, only the 12.9 year eccentric orbit gives a reasonably large mass sum of $2 \mathcal{M}_{\odot}$. Its major axis is oriented toward us, the inclination is $99^{\circ}$, hence the apparent separation is always less than the true semimajor axis. The elements $e$ and $i$ are strongly correlated. Interferometric and RV monitoring of the next periastron in 2023.6 is needed.
The orbit predicts the RV difference of $\sim 30 \mathrm{~km} \mathrm{~s}^{-1}$ near the periastron.

17447-4244. We reprocessed the observations of FIN 341 made at SOAR in 2008 and 2009 to verify that the quadrant was opposite to that of 2015-2017, in agreement with the proposed 15 year orbit. One of the two archival speckle measures made in 1989.3 fits the orbit nicely, while the separation of 0 '! 15 measured on 1991.39 strongly contradicts both the orbit (which predicts the separation of 20 mas) and the non-resolution by Hipparcos. We discard the 1991.39 speckle datum and use the interferometric measures by W. Finsen made in 1959 and 1963. This object belongs to the $\lambda$ Bootis class of chemically peculiar stars (Paunzen et al. 1997), making the measurement of its mass particularly interesting. The masses of 1.9 and $1.5 \mathcal{M}_{\odot}$ deduced from the orbit are slightly less than expected for normal A2V stars.

$18040+0150$. This is HD 165045, a double-lined spectroscopic binary resolved at SOAR. The RVs measured by D. Latham (2012, private communication) are used together with the speckle measures in the combined orbit with $P=1.6$ year. Its small inclination prevents accurate measurement of the masses; the orbital parallax is $29.2 \pm 3.4$ mas.

18520-5418. The Hipparcos astrometric binary was first resolved at Gemini in 2012 by Tokovinin et al. (2013) and then followed at SOAR. The period of this preliminary orbit is fixed to 8 years. One can't help noting the discrepancy between the Gaia parallax of 13.77 mas, the HIP2 parallax of 19.5 mas, and the dynamical parallax of 21.5 mas. The Gaia parallax leads to the unrealistically large mas sum of $6.2 \mathcal{M}_{\odot}$ and is therefore suspect. With a physical companion at $146^{\prime \prime}$, the system is triple.

19377-4128. Both extremities of the 55 year circular orbit of VOU 34 are covered by accurate speckle measures. The pair has made 1.5 revolutions since its first resolution in 1936 . The moderate $\Delta V \approx 0.4 \mathrm{mag}$ and the opposite quadrants in 1991 (Hipparcos) and 2015 exclude the alternative eccentric orbit with half the period.

19598-095\%. The combined 5 year orbit by Pourbaix (2002) had large residuals to the SOAR measures, prompting its revision. The new combined orbit uses the published RVs and is well defined (grade 2). Its small inclination and correspondingly small RV amplitudes do not help accurate measurement of the masses and orbital parallax, which is $40.0 \pm 5.8$ mas.

20212-514\%. The Hipparcos binary HDS 2097 is quite fast, with $P=10$ years and a well-defined first orbit. The star is on the Magellan program of exo-planet search (Arrigada 2011)

21099-2424. The first 6.9 year orbit of HIP 104476 is well defined by the SOAR data. SIMBAD calls this star "pre-main sequence"; it is an X-ray source. The components are located on the main sequence, however.

21368-3043. The preliminary orbit of VOU 35 by Tokovinin et al. (2014) is revised here using recent observations. The pair has a relatively close companion $\mathrm{C}$, discovered at SOAR at 0". 45 from $\mathrm{A}$ and showing rapid orbital motion (the estimated period of $\mathrm{AB}, \mathrm{C}$ is $\sim 100$ years).

21400-5222. The SOAR data cover the same part of the 21 year orbit where the pair was resolved by Hipparcos one revolution earlier. The new orbit is preliminary. 
The components are nearly equal; both are located on the subgiant branch in the CMD in Figure 2, in agreement with the F7III spectral type.

21504-5818. The first 17 year orbit of HIP 107806 is well covered. The mass sum computed with the Hipparcos parallax of 24.1 mas is $1.4 \mathcal{M}_{\odot}$, appropriate for the G6V spectral type. The dynamical parallax in Table 3 is smaller and the derived masses are correspondingly larger.

22228-2937. HDS 3172 is a fast pair of G6V dwarfs with $P=10.5$ years. Its first orbit (Figure 1) is well constrained. The resolution in 2016.9, at periastron (38 mas), is tentative. Now the pair opens up again.

22535-113\%. This is the bright triple system HR 8704 (74 Aqr) where the primary component of the interferometric pair A,B (MCA 73) is a 3.4 day double-lined spectroscopic binary (Catanzaro \& Leto 2004). The 19 year outer orbit was first computed in 1993 and updated several times since, the last one by Mason et al. (2010). This is a typical case where the measurements can be modeled either by a highly inclined orbit with moderate eccentricity (the 19 year orbit) or by a very eccentric orbit with half the period. The ambiguity can be resolved when the true quadrants are known, which is not the case for binaries with small $\Delta m$ and for classical speckle observations. The quadrants of MCA 73 with $\Delta m \approx 0.6 \mathrm{mag}$ can be determined from the speckle image reconstruction which is used at SOAR since 2015. We found that in 2015 and 2016 the quadrants differed from the orbit prediction, whereas they matched in 2008.76. This archival observation was reprocessed to confirm the quadrant; see also the adaptive optics measurement by Schoeller et al. (2010). Therefore, the 19 year orbit is wrong, the pair is always resolved in the same quadrant. The 9.5 year orbit with $e=0.86$ is computed here. Catanzaro \& Leto (2004) give the RVs of the center of mass of the close pair (component A) and one measurement of the secondary B. These RVs support the eccentric orbit (Figure 3) which predicts large RV variation near the periastron. The RV amplitudes are $K_{1}=10.0 \mathrm{~km} \mathrm{~s}^{-1}$ and $K_{2}=19.6 \mathrm{~km} \mathrm{~s}^{-1}$, the systemic velocity is $-2.7 \mathrm{~km} \mathrm{~s}^{-1}$. The $\mathrm{RV}$ amplitudes and inclination correspond to the mass sum of $\sim 15 \mathcal{M}_{\odot}$; however, the large error of the inclination, $i=30^{\circ} \pm 17^{\circ}$, makes the spectroscopic masses quite uncertain. The HIP2 parallax of 3.95 mas, also not very precise, leads to the mass sum of $16.6 \mathcal{M}_{\odot}$. The orbit thus roughly matches the estimated mass sum of three B9V stars, 11 $\mathcal{M}_{\odot}$.

This chemically peculiar HgMn star has attracted considerable attention (135 references in SIMBAD). Spectroscopic and interferometric coverage of the next periastron in 2019.5 may lead to the more accurate measurements of the masses and the distance, especially if the closest approach at 6 mas can be resolved with long-baseline interferometers. The semimajor axis of the inner spectroscopic pair is 0.3 mas.

\section{SUMMARY}

Speckle monitoring of close visual binaries, started at SOAR in 2008, begins to bear fruits by defining previously unknown orbital elements. Many southern binaries discovered by Hipparcos had not been followed and made one or more revolutions before their orbits could be determined. Although some orbits are now well constrained, there are still many binaries with sparse coverage where more observations are needed. Their short (on the order of a decade) periods mean that this work can be accomplished in several years if the speckle program at SOAR continues.

We feel that the knowledge of orbits will be critical for correct interpretation of the Gaia astrometry. As a reward, a large set of accurately measured stellar masses will become available. Apart from many common binaries composed of two normal dwarfs, there will be interesting exceptions such as massive or evolved stars, members of clusters and hierarchical multiple systems, stars with peculiar spectra, unusual chemical composition, or young age. Accurate measurements of their masses will be of great value.

The detailed report by the Referee, B. Mason, who checked the orbits and assigned the grades, helped to improve this paper. This work used the SIMBAD service operated by Centre des Données Stellaires (Strasbourg, France), bibliographic references from the Astrophysics Data System maintained by SAO/NASA, and the Washington Double Star Catalog maintained at USNO.

Facilities: SOAR.

\section{REFERENCES}

Abt, H. A. \& Willmarth, D. W. 1999, ApJ, 521, 682

Arrigada, P. 2011, ApJ, 734, 70

Baraffe, I., Homeier, D., Allard, F. \& Chabrier, G. 2015, A\&A, 577, 42 (BHAC15)

Catanzaro, G. \& Leto, P. 2004, A\&A, 416, 661

Cvetkovic, Z., Pavlovic, R. \& Ninkovic, S. 2016 AJ, 151, 83

Cvetkovic, Z. 2013, IAU Inf. Circ. 181, 1

Docobo, J. A., Griffin, R. F., Campo, P. P., \& Abushattal, A. A. 2017, MNRAS, 469, 1096

Dotter, A., Chaboyer, B., Jevremović, D. et al. 2008, ApJS, 178, 89

Ehrenreich, D., Lagrange, A.-M., Montagnier, G. et al. 2010, A\&A, 523, 73

Griffin, R. F. 2000, Obs., 120, 1

Griffin, R. F. 2012, JAA, 33, 29

Griffin, R. F. 2015, Obs, 135, 321

Hartkopf, W. I., Mason, B. D. \& Worley, C. E. 2001, AJ, 122, 3472 (VB6)

Hartkopf, W. I., Mason, B. D., \& McAlister, H. A. 2001, AJ, 122, 3480

Hartkopf, W. I., Tokovinin, A. \& Mason, B. D. 2012, AJ, 143, 42

Heintz, W. D. 1996, AJ, 111, 408

Horch, E. P., van Belle, G. T., Davidson, J. W., Jr. et al. 2015, AJ, 150,151

Horch, E. P., Casetti-Dinescu, D. I., Camarata, M. A. et al. 2017, AJ, 153,212

Hutchings, J. B., Griffin, R. F. \& Menard, F. 2000, PASP, 112, 833

Jenkins, J. S., Diaz, M., Jones, H. R. A. et al. 2015, MNRAS, 453, 1439

Lindegren, L., Lammers, U., Bastian, U. et al. 2016, A\&A, 595, 4

Mason, B. D., Wycoff, G. L., Hartkopf, W. I. et al. 2001, AJ, 122, 3466 (WDS)

Mason, B. D., Hartkopf, W. I., \& Tokovinin, A. 2010, AJ, 140, 735

Nordström, B., Mayor, M., Andersen, J. et al. 2004, A\&A, 418, 989

Olevic, D. \& Jovanovic, P. 1998, Serbian AJ, 158, 73,

Olevic, D. 2002, IAU Inf. Circ. 146, 1

Paunzen, E., Weiss, W. W., Heiter, U. \& North, P. 1997, A\&AS, 123,93

Pourbaix, D. 2000, A\&A, 145, 215

Riddle, R. L., Tokovinin, A., Mason, B. D. et al. 2015, ApJ, 799, 4

Schoeller, M., Correia, S., Hubrig, S. \& Ageorges, N. 2010, A\&A, 522, 85 
Shatskii, N. I. \& Tokovinin, A. A. 1009, AstL, 24, 673

Söderhjelm, S. 1999, A\&A, 341, 121

Tokovinin, A., Mason, B. D., \& Hartkopf, W. I. 2010a, AJ, 139, 743

Tokovinin, A. Cantarutti, R., Tighe, R. et al. 2010b, PASP, 122, 1483

Tokovinin, A. 2012, AJ, 144, 56 (Tok2012b)

Tokovinin, A., Hartung, M., \& Hayward, Th. L. 2013, AJ, 146, 8

Tokovinin, A., Mason, B. D., \& Hartkopf, W. I. 2014, AJ, 147, 123
Tokovinin, A., Mason, B. D., \& Hartkopf, W. I. et al. 2015, AJ, 150,50

Tokovinin, A., Mason, B. D., \& Hartkopf, W. I. et al. 2016, AJ, 153,151

Tokovinin, A. 2016, AJ, 152, 138 (Paper I).

van Leeuwen, F. 2007, A\&A, 474, 653 (HIP2) 
Table 1

Orbital Elements

\begin{tabular}{|c|c|c|c|c|c|c|c|c|c|c|}
\hline $\begin{array}{l}\text { WDS } \\
\text { HIP }\end{array}$ & $\begin{array}{c}\text { Discoverer } \\
\text { Designation }\end{array}$ & $\begin{array}{c}P \\
(\mathrm{yr})\end{array}$ & $\begin{array}{l}T_{0} \\
(\mathrm{yr})\end{array}$ & $e$ & $\begin{array}{c}a \\
\left({ }^{\prime \prime}\right)\end{array}$ & $\begin{array}{l}\Omega \\
\left({ }^{\circ}\right)\end{array}$ & $\begin{array}{l}\omega \\
\left(^{\circ}\right)\end{array}$ & $\begin{array}{c}i \\
\left({ }^{\circ}\right)\end{array}$ & $\mathrm{Gr}$ & $\begin{array}{c}\text { Orbit } \\
\text { Reference }\end{array}$ \\
\hline $\begin{array}{c}00135-3650 \\
1083\end{array}$ & HDS 32 & $\begin{array}{l}15.34 \\
\pm 0.35\end{array}$ & $\begin{array}{r}2009.45 \\
\pm 0.73\end{array}$ & $\begin{array}{r}0.25 \\
\pm 0.08\end{array}$ & $\begin{array}{r}0.229 \\
\pm 0.016\end{array}$ & $\begin{array}{r}88.2 \\
\pm 48.5\end{array}$ & $\begin{array}{r}260.6 \\
\pm 38.4\end{array}$ & $\begin{array}{l}154.2 \\
\pm 11.3\end{array}$ & 3 & $\mathrm{New}^{\mathrm{b}}$ \\
\hline $\begin{array}{c}00164-7024 \\
1309\end{array}$ & HEI 198 & $\begin{array}{r}63.3 \\
+30.8\end{array}$ & $\begin{array}{r}2010.7 \\
+2.7\end{array}$ & $\begin{array}{r}0.42 \\
+0.15\end{array}$ & $\begin{array}{r}0.166 \\
+0.072\end{array}$ & $\begin{array}{r}153.2 \\
+10.6\end{array}$ & $\begin{array}{r}210.0 \\
+25.5\end{array}$ & $\begin{array}{r}121.6 \\
+15.0\end{array}$ & 5 & New $^{b}$ \\
\hline $\begin{array}{c}00258+1025 \\
2035\end{array}$ & HDS 57 & $\begin{array}{r}48.87 \\
+7.32\end{array}$ & $\begin{array}{r}2020.24 \\
+0.60\end{array}$ & $\begin{array}{r}0.105 \\
\text { fixed }\end{array}$ & $\begin{array}{r}0.149 \\
+0.015\end{array}$ & $\begin{array}{r}10.0 \\
28.2 \\
+81\end{array}$ & $\begin{array}{r}72.2 \\
+3.7\end{array}$ & $\begin{array}{r}11.0 \\
123.2 \\
+19\end{array}$ & 4 & New \\
\hline $01334-4354$ & HDS 205 & 7.766 & 2011.025 & 0.493 & 0.1308 & $\begin{array}{l}10.1 \\
198.0\end{array}$ & 115.5 & 65.5 & 2 & $\mathrm{New}^{\mathrm{a}}$ \\
\hline 7254 & & \pm 0.041 & \pm 0.014 & \pm 0.012 & \pm 0.0013 & \pm 0.8 & \pm 1.1 & \pm 0.6 & & \\
\hline $\begin{array}{c}02166-5026 \\
10611\end{array}$ & TOK 185 & $\begin{array}{r}13.97 \\
\pm 4.44\end{array}$ & $\begin{array}{r}2013.89 \\
\pm 0.25\end{array}$ & $\begin{array}{r}0.20 \\
\pm 0.19\end{array}$ & $\begin{array}{r}0.1038 \\
\pm 0.0247\end{array}$ & $\begin{array}{r}276.8 \\
\pm 8.0\end{array}$ & $\begin{array}{r}15.8 \\
\pm 10.4\end{array}$ & $\begin{array}{r}50.0 \\
\pm 7.2\end{array}$ & 4 & Tok2015c \\
\hline $\begin{array}{c}02390-5811 \\
12351\end{array}$ & HDS 345 & $\begin{array}{r}6.019 \\
\pm 0.020\end{array}$ & $\begin{array}{r}2016.894 \\
\pm 0.007\end{array}$ & $\begin{array}{r}0.516 \\
\pm 0.011\end{array}$ & $\begin{array}{r}0.1976 \\
\pm 0.0026\end{array}$ & $\begin{array}{r}257.5 \\
\pm 3.2\end{array}$ & $\begin{array}{r}94.5 \\
\pm 2.5\end{array}$ & $\begin{array}{r}138.1 \\
\pm 1.3\end{array}$ & 3 & New $^{b}$ \\
\hline $04007+2023$ & TOK $363 \mathrm{Aa}, \mathrm{Ab}$ & 16.761 & 2010.259 & 0.311 & 0.1141 & 198.2 & 146.5 & 31.5 & $5(3)$ & Gri2012 \\
\hline 18719 & & \pm 0.115 & \pm 0.191 & \pm 0.014 & \pm 0.0030 & \pm 3.0 & \pm 4.2 & \pm 4.4 & & \\
\hline $04049-3527$ & CHR 224 BC & $\begin{array}{r}52.17 \\
+2.25\end{array}$ & 2009.67 & 0.920 & $\begin{array}{r}0.2714 \\
+0.004\end{array}$ & 85.4 & 153.6 & 123.2 & 4 & $\mathrm{New}^{\mathrm{a}}$ \\
\hline $\begin{array}{c}19052 \\
04074-6413\end{array}$ & LMP 51 & $\begin{array}{r} \pm 2.35 \\
26.028\end{array}$ & $\begin{array}{r} \pm 0.07 \\
1996.458\end{array}$ & $\begin{array}{r} \pm 0.006 \\
0.57\end{array}$ & $\begin{array}{r} \pm 0.0042 \\
0.4201\end{array}$ & $\begin{array}{r} \pm 1.9 \\
294.8\end{array}$ & $\begin{array}{r} \pm 3.0 \\
221.0\end{array}$ & $\begin{array}{r} \pm 1.5 \\
96.5\end{array}$ & $5(4)$ & Jen2015 \\
\hline 19233 & & $\begin{array}{l}20.020 \\
\pm 0.076\end{array}$ & $\begin{array}{r}190.400 \\
\text { fixed }\end{array}$ & fixed & \pm 0.0067 & $\begin{array}{r}28.0 \\
\pm 1.1\end{array}$ & fixed & $\begin{array}{l}90.5 \\
\pm 0.5\end{array}$ & $0(4)$ & Jen_U15 \\
\hline $04107-0452$ & A 2801 & 20.621 & 2014.031 & 0.8400 & 0.1672 & 153.3 & 68.6 & 69.0 & $2(1)$ & Tok2014a,Gri2015 \\
\hline 19508 & & \pm 0.005 & \pm 0.003 & \pm 0.0014 & \pm 0.0021 & \pm 0.6 & \pm 0.3 & \pm 0.5 & & \\
\hline $04422+0259$ & A 2424 & 58.1 & 2011.05 & 0.345 & 0.1545 & 228.6 & 265.1 & 85.3 & 3 & Tok2015c \\
\hline 21880 & & \pm 4.8 & \pm 0.63 & \pm 0.026 & \pm 0.0085 & \pm 0.8 & \pm 8.3 & \pm 0.6 & & \\
\hline $05086-1810$ & WSI 72 & 0.962 & 2016.174 & 0.258 & 0.0994 & 39.8 & 163.2 & 93.8 & 2 & $\mathrm{New}^{\mathrm{a}}$ \\
\hline 23932 & & \pm 0.002 & \pm 0.016 & \pm 0.025 & \pm 0.0022 & \pm 0.4 & \pm 4.8 & \pm 0.7 & & \\
\hline $05103-0736$ & A 484 & 18.90 & 1999.74 & 0.817 & 0.1719 & 290.3 & 123.2 & 104.2 & 3 & Hrt2012a \\
\hline 24076 & & \pm 0.07 & \pm 0.39 & \pm 0.031 & \pm 0.0183 & \pm 1.2 & \pm 6.2 & \pm 2.2 & & \\
\hline $05525-0217$ & HDS 787 & 11.963 & 1999.710 & 0.244 & 0.1207 & 331.1 & 271.8 & 57.2 & 2 & Tok2014a \\
\hline 27758 & & \pm 0.036 & \pm 0.045 & \pm 0.005 & \pm 0.0007 & \pm 0.6 & \pm 0.7 & \pm 0.5 & & \\
\hline $06454-3148$ & EHR $9 \mathrm{Ba}, \mathrm{Bb}$ & 6.909 & 2014.458 & 0.206 & 0.1173 & 193.6 & 349.5 & 140.6 & 3 & Tok2015c \\
\hline $32366 \mathrm{~B}$ & & \pm 0.051 & \pm 0.066 & \pm 0.016 & \pm 0.0032 & \pm 4.1 & \pm 4.5 & \pm 2.6 & & \\
\hline $06533-1902$ & CHR 169 & 36.28 & 2017.64 & 0.533 & 0.1928 & 353.7 & 257.8 & 105.3 & 3 & New \\
\hline 33077 & & \pm 0.82 & \pm 0.12 & \pm 0.033 & \pm 0.0049 & \pm 1.0 & \pm 1.5 & \pm 1.3 & & \\
\hline $07269+2015$ & CHR 26 & 8.571 & 2000.494 & 0.412 & 0.0518 & 341.4 & 246.7 & 50.1 & 2 & Ole1998c \\
\hline 36156 & & \pm 0.068 & \pm 0.113 & \pm 0.068 & \pm 0.0025 & \pm 5.7 & \pm 6.7 & \pm 5.0 & & \\
\hline $08122+1739$ & $\mathrm{HUT} 1 \mathrm{Ca}, \mathrm{Cb}$ & 17.263 & 1997.743 & 0.180 & 0.3592 & 81.0 & 287.3 & 150.0 & $4(3)$ & RAO2015,Gri2000 \\
\hline 40167 & & \pm 0.032 & \pm 0.160 & \pm 0.013 & \pm 0.0058 & \pm 1.9 & \pm 3.6 & fixed & & \\
\hline $08403+1921$ & CHR 130 & 35.45 & 1987.86 & 0.169 & 0.1402 & 339.8 & 226.3 & 90.4 & 3 & Ole2002b \\
\hline 42542 & & \pm 0.22 & \pm 0.53 & \pm 0.013 & \pm 0.0021 & \pm 0.6 & \pm 5.8 & \pm 0.6 & & \\
\hline $08447-4238$ & CHR 238 & 2.262 & 2012.584 & 0.676 & 0.0775 & 13.0 & 125.2 & 152.2 & 4 & Tok2015c \\
\hline 42916 & & \pm 0.001 & \pm 0.007 & \pm 0.007 & \pm 0.0015 & \pm 5.2 & \pm 5.1 & \pm 3.8 & & \\
\hline $09252-1258$ & WSI 73 & 13.15 & 2018.94 & 0.61 & 0.1443 & 93.6 & 52.2 & 87.0 & 3 & Tok2015a \\
\hline 46191 & & \pm 0.61 & \pm 1.40 & fixed & \pm 0.0304 & \pm 1.5 & \pm 19.7 & \pm 1.5 & & \\
\hline $10121-0241$ & DEL 3 & 6.365 & 2015.502 & 0.50 & 0.2624 & 249.7 & 94.7 & 91.8 & 4 & New \\
\hline 49969 & & \pm 0.027 & \pm 0.030 & fixed & \pm 0.0051 & \pm 0.6 & \pm 0.8 & \pm 0.4 & & \\
\hline $10294+1211$ & HDS 1507 & 15.59 & 2011.79 & 0.372 & 0.0984 & 277.0 & 286.3 & 24.6 & 3 & Cve2016b ${ }^{b}$ \\
\hline 51360 & & \pm 0.11 & \pm 0.36 & \pm 0.032 & \pm 0.0021 & \pm 29.7 & \pm 24.7 & \pm 6.9 & & \\
\hline $10529-1717$ & HDS 1556 & 14.95 & 2003.60 & 0.553 & 0.1875 & 109.3 & 61.8 & 97.0 & 2 & Tok2015c \\
\hline $\begin{array}{c}53206 \\
11420-1701\end{array}$ & TOK 384 Aa Ab & \pm 0.26 & $\begin{array}{r} \pm 0.22 \\
2016.21\end{array}$ & $\begin{array}{r} \pm 0.032 \\
0.58\end{array}$ & \pm 0.0056 & $\begin{array}{r} \pm 0.3 \\
169.0\end{array}$ & \pm 2.3 & \pm 0.5 & 5 & New ${ }^{b}$ \\
\hline $\begin{array}{c}11420-1801 \\
57078\end{array}$ & & $\begin{array}{r}21.1 \\
\pm 8.7\end{array}$ & $\begin{array}{r}2010.21 \\
\pm 0.10\end{array}$ & $\begin{array}{r}0.08 \\
\pm 0.12\end{array}$ & $\begin{array}{r}0.103 \\
\pm 0.040\end{array}$ & $\begin{array}{r}109.0 \\
\pm 40.5\end{array}$ & $\begin{array}{r}259.0 \\
\pm 46.5\end{array}$ & $\begin{array}{r}21.1 \\
\pm 5.4\end{array}$ & 5 & New ${ }^{\circ}$ \\
\hline $12485-1543$ & WSI $74 \mathrm{Aa}, \mathrm{Ab}$ & 2.661 & 2011.094 & 0.508 & 0.0843 & 146.6 & 145.6 & 55.3 & 2 & Tok2014a ${ }^{\mathrm{c}}$ \\
\hline 62505 & & \pm 0.002 & \pm 0.005 & \pm 0.007 & \pm 0.0008 & \pm 0.9 & \pm 1.3 & \pm 0.8 & & \\
\hline $13137-6248$ & HDS 1852 & 25.644 & 1996.680 & 0.291 & 0.1659 & 149.1 & 124.0 & 63.1 & 5 & $\mathrm{New}^{\mathrm{b}}$ \\
\hline 64537 & & 0.401 & & \pm 0.068 & \pm 0.0059 & & \pm 15.0 & \pm 1.9 & & \\
\hline $14330-4224$ & HDS 2054 & 66.5 & 1997.84 & 0.80 & 0.207 & 356.7 & 116.0 & 97.5 & 4 & New \\
\hline 71140 & & \pm 22.2 & & fixed & \pm 0.049 & & \pm 6.0 & \pm 1.6 & & \\
\hline $15245-1322$ & HDS 2167 & 82.30 & 2008.37 & 0.422 & 0.440 & 303.5 & 157.1 & 108.9 & 4 & New \\
\hline 75416 & & \pm 10.11 & & \pm 0.049 & \pm 0.037 & & & \pm 0.6 & & \\
\hline $15339-1700$ & HDS 2185 & 72.32 & 2010.26 & 0.414 & 0.5757 & 150.6 & 310.3 & 34.6 & 4 & Tok2012b \\
\hline 76203 & & \pm 2.20 & \pm 0.10 & \pm 0.012 & \pm 0.0069 & \pm 1.1 & \pm 1.1 & \pm 1.0 & & \\
\hline $17176+1025$ & HDS 2445 & 45.80 & 2009.14 & 0.149 & 0.2781 & 84.7 & 140.4 & 97.2 & 3 & Cve2013b \\
\hline & & & & & \pm 0.0063 & & \pm 23.7 & \pm 0.5 & & \\
\hline $17195-5004$ & FIN 356 & 12.93 & 2010.65 & 0.814 & 0.114 & 85.9 & 93.6 & 98.6 & 2 & New \\
\hline & & & & & \pm 0.016 & & \pm 1.4 & \pm 1.9 & & \\
\hline $17447-4244$ & FIN 341 & 15.12 & 2012.71 & 0.165 & 0.1183 & 179.5 & 244.6 & 81.7 & 5 & New \\
\hline 86847 & & \pm 0.10 & & \pm 0.038 & \pm 0.0049 & \pm 0.8 & \pm 14.4 & \pm 1.6 & & \\
\hline $18040+0150$ & TOK 695 & 1.615 & 1996.698 & 0.501 & 0.0472 & 271.0 & 287.4 & 37.7 & $4(3)$ & $\mathrm{New}^{\mathrm{c}}$ \\
\hline 88481 & & \pm 0.001 & & \pm 0.011 & \pm 0.0016 & \pm 2.5 & \pm 2.5 & \pm 3.8 & & \\
\hline $18520-5418$ & TOK $325 \mathrm{Aa}, \mathrm{Ab}$ & 8.0 & 2017.985 & 0.413 & 0.1012 & 92.4 & 41.2 & 60.8 & 4 & New \\
\hline 92592 & & fixed & \pm 0.136 & \pm 0.029 & \pm 0.0029 & \pm 2.5 & \pm 4.6 & \pm 2.0 & & \\
\hline $19377-4128$ & VOU 34 & 55.0 & 2003.75 & 0.02 & 0.1642 & 134.4 & 86.0 & 96.9 & 3 & New \\
\hline 96545 & & \pm 6.8 & \pm 4.40 & \pm 0.12 & \pm 0.0045 & \pm 0.6 & \pm 29.4 & \pm 1.1 & & \\
\hline $19598-0957$ & HO 276 & 4.867 & 2012.022 & 0.605 & 0.1514 & 326.6 & 319.4 & 18.8 & 2 & $\mathrm{Pbx} 2000 \mathrm{~b}^{\mathrm{c}}$ \\
\hline 98416 & & \pm 0.004 & \pm 0.011 & \pm 0.004 & \pm 0.0013 & \pm 1.8 & \pm 2.2 & \pm 2.7 & & \\
\hline
\end{tabular}


Table 1 - Continued

\begin{tabular}{|c|c|c|c|c|c|c|c|c|c|c|}
\hline $\begin{array}{l}\text { WDS } \\
\text { HIP }\end{array}$ & $\begin{array}{l}\text { Discoverer } \\
\text { Designation }\end{array}$ & $\begin{array}{c}P \\
(\mathrm{yr})\end{array}$ & $\begin{array}{l}T_{0} \\
(\mathrm{yr})\end{array}$ & $e$ & $\begin{array}{c}a \\
\left({ }^{\prime \prime}\right)\end{array}$ & $\begin{array}{c}\Omega \\
\left(^{\circ}\right)\end{array}$ & $\begin{array}{l}\omega \\
\left(^{\circ}\right)\end{array}$ & $\begin{array}{c}i \\
\left(^{\circ}\right)\end{array}$ & Gr & $\begin{array}{c}\text { Orbit } \\
\text { Reference }\end{array}$ \\
\hline $\begin{array}{c}20212-5147 \\
100356\end{array}$ & HDS 2907 & $\begin{array}{r}9.886 \\
\pm 0.129\end{array}$ & $\begin{array}{r}2017.514 \\
\pm 0.016\end{array}$ & $\begin{array}{r}0.651 \\
\pm 0.016\end{array}$ & $\begin{array}{r}0.1948 \\
\pm 0.0047\end{array}$ & $\begin{array}{r}32.7 \\
\pm 3.2\end{array}$ & $\begin{array}{r}194.0 \\
\pm 5.5\end{array}$ & $\begin{array}{r}48.8 \\
\pm 2.3\end{array}$ & 3 & New \\
\hline $\begin{array}{c}21099-2424 \\
104476\end{array}$ & HDS 3015 & $\begin{array}{r}6.88 \\
\pm 0.12\end{array}$ & $\begin{array}{r}2014.99 \\
\pm 0.18\end{array}$ & $\begin{array}{r}0.566 \\
\pm 0.032\end{array}$ & $\begin{array}{r}0.0882 \\
\pm 0.0040\end{array}$ & $\begin{array}{r}296.3 \\
\pm 4.5\end{array}$ & $\begin{array}{r}13.7 \\
\pm 12.2\end{array}$ & $\begin{array}{r}66.0 \\
\pm 2.0\end{array}$ & 2 & New $^{\mathrm{a}}$ \\
\hline $\begin{array}{c}21368-3043 \\
106701\end{array}$ & VOU 35 & $\begin{array}{r}19.547 \\
\pm 0.086\end{array}$ & $\begin{array}{r}2018.160 \\
\pm 0.088\end{array}$ & $\begin{array}{r}0.390 \\
\pm 0.033\end{array}$ & $\begin{array}{r}0.1673 \\
\pm 0.0037\end{array}$ & $\begin{array}{r}126.9 \\
\pm 1.2\end{array}$ & $\begin{array}{r}91.4 \\
\pm 1.4\end{array}$ & $\begin{array}{r}107.8 \\
\pm 0.8\end{array}$ & 3 & Tok2014a \\
\hline $\begin{array}{c}21400-5222 \\
106978\end{array}$ & HDS 3084 & $\begin{array}{r}21.31 \\
\pm 0.74\end{array}$ & $\begin{array}{r}1998.80 \\
\pm 1.45\end{array}$ & $\begin{array}{r}0.54 \\
\pm 0.18\end{array}$ & $\begin{array}{r}0.0762 \\
\pm 0.0084\end{array}$ & $\begin{array}{r}31.5 \\
\pm 74.2\end{array}$ & $\begin{array}{r}231.4 \\
\pm 66.6\end{array}$ & $\begin{array}{l}150.4 \\
\pm 11.1\end{array}$ & 3 & $\mathrm{New}^{\mathrm{b}}$ \\
\hline $\begin{array}{c}21504-5818 \\
107806\end{array}$ & HDS 3109 & $\begin{array}{r}16.79 \\
\pm 0.75\end{array}$ & $\begin{array}{r}2004.15 \\
\pm 0.46\end{array}$ & $\begin{array}{r}0.246 \\
\pm 0.062\end{array}$ & $\begin{array}{r}0.1782 \\
\pm 0.0039\end{array}$ & $\begin{array}{r}307.5 \\
\pm 0.4\end{array}$ & $\begin{array}{r}255.3 \\
\pm 3.7\end{array}$ & $\begin{array}{r}86.9 \\
\pm 0.5\end{array}$ & 3 & New \\
\hline $\begin{array}{c}22228-2937 \\
110483\end{array}$ & HDS 3172 & $\begin{array}{l}10.51 \\
\pm 0.25\end{array}$ & $\begin{array}{r}2017.374 \\
\pm 0.069\end{array}$ & $\begin{array}{r}0.288 \\
\pm 0.023\end{array}$ & $\begin{array}{r}0.1444 \\
\pm 0.0031\end{array}$ & $\begin{array}{r}155.4 \\
\pm 0.4\end{array}$ & $\begin{array}{r}325.7 \\
\pm 3.1\end{array}$ & $\begin{array}{l} \pm 0.0 \\
97.4 \\
\pm 0.5\end{array}$ & 2 & $\mathrm{New}^{\mathrm{a}}$ \\
\hline $\begin{array}{c}22535-1137 \\
113031\end{array}$ & MCA 73 & $\begin{array}{r}9.479 \\
\pm 0.044\end{array}$ & $\begin{array}{r}2010.039 \\
\pm 0.134\end{array}$ & $\begin{array}{r}0.862 \\
\pm 0.029\end{array}$ & $\begin{array}{r}0.0460 \\
\pm 0.0061\end{array}$ & $\begin{array}{r}40.9 \\
\pm 20.3\end{array}$ & $\begin{array}{r}70.7 \\
\pm 16.1\end{array}$ & $\begin{array}{r}29.8 \\
\pm 17.4\end{array}$ & 2 & Msn2010c \\
\hline
\end{tabular}

References. - Cve2013b - Cvetkovid (2013); Cve2016b - Cvetkovic et al. (2016); Jen2015 - Jenkins et al. (2015); Gri2000 - Griffin (2000); Gri2012 - Griffin (2012); Gri2015 - Griffin (2015); Hor2017 - Horch et al. (2017); Hrt2012a - Hartkopf et al. (2012); Msn2010c - Mason et al. (2010); Ole1998c - Olevic \& Jovanovic (1998); Ole2002b - Olevic (2002); Pbx2000b - Pourbaix (2002); RAO2015 - Riddle et al. (2015); Tok2012b - Tokovinin (2012); Tok2014a - Tokovinin et al. (2014); Tok2015c - Tokovinin et al. (2015).

a See Figure 1.

b Insufficient coverage.

${ }^{\mathrm{c}}$ Combined orbit using the RVs.

Table 2

Observations and residuals (Fragment)

\begin{tabular}{cccccrrr}
\hline \hline WDS & $\begin{array}{c}T \\
(\mathrm{yr})\end{array}$ & $\begin{array}{c}\theta \\
\left({ }^{\circ}\right)\end{array}$ & $\begin{array}{c}\rho \\
\left({ }^{\prime \prime}\right)\end{array}$ & $\begin{array}{c}\sigma \\
\left({ }^{\prime \prime}\right)\end{array}$ & $\begin{array}{c}\mathrm{O}-\mathrm{C}_{\theta} \\
\left({ }^{\circ}\right)\end{array}$ & $\begin{array}{c}\mathrm{O}-\mathrm{C}_{\rho} \\
\left({ }^{\prime \prime}\right)\end{array}$ & Flag \\
\hline $00135-3650$ & 1991.2500 & 282.0 & 0.2190 & 0.0100 & 0.0 & -0.0000 & $\mathrm{H}$ \\
$00135-3650$ & 2014.7662 & 46.3 & 0.2565 & 0.0030 & -0.0 & -0.0000 & $\mathrm{~S}$ \\
$00135-3650$ & 2015.9130 & 28.0 & 0.2600 & 0.0030 & 0.1 & -0.0000 & $\mathrm{~S}$ \\
$00135-3650$ & 2016.9506 & 11.3 & 0.2592 & 0.0030 & -0.0 & 0.0001 & $\mathrm{~S}$ \\
\hline
\end{tabular}


Table 3

Parallaxes and photometry

\begin{tabular}{|c|c|c|c|c|c|c|c|c|c|c|c|c|}
\hline WDS & HIP & $\begin{array}{l}\text { Spectral } \\
\text { Type }\end{array}$ & $\begin{array}{c}\pi_{\mathrm{HIP} 2} \\
(\mathrm{mas})\end{array}$ & $\begin{array}{c}\pi_{\text {dyn }} \\
(\text { mas })\end{array}$ & $\begin{array}{c}\mathcal{M}_{1} \\
\left(\mathcal{M}_{\odot}\right)\end{array}$ & $\begin{array}{c}\mathcal{M}_{2} \\
\left(\mathcal{M}_{\odot}\right)\end{array}$ & $\begin{array}{c}V \\
(\mathrm{mag})\end{array}$ & $\begin{array}{c}V-I_{C} \\
(\mathrm{mag})\end{array}$ & $\begin{array}{c}\Delta y \\
(\mathrm{mag})\end{array}$ & $\begin{array}{c}\sigma_{\Delta y} \\
(\mathrm{mag})\end{array}$ & $\begin{array}{c}\Delta I \\
(\mathrm{mag})\end{array}$ & $\begin{array}{c}\sigma_{\Delta I} \\
(\mathrm{mag})\end{array}$ \\
\hline $00135-3650$ & 1083 & $\mathrm{M} 0.5 \mathrm{~V}$ & 35.5 & $36.0^{*}$ & 0.59 & 0.52 & 10.77 & 1.78 & & & 0.77 & 0.06 \\
\hline $00164-7024$ & 1309 & F0V & 5.2 & 6.9 & 1.84 & 1.61 & 7.18 & 0.28 & 0.68 & $\ldots$ & 0.55 & 0.02 \\
\hline $00258+1025$ & 2035 & G0 & 9.1 & 8.7 & 1.19 & 0.93 & 9.21 & 0.71 & & & 1.04 & 0.21 \\
\hline $01334-4354$ & 7254 & $\mathrm{~K} 0 / \mathrm{K} 1 \mathrm{~V}$ & 26.3 & $27.5^{*}$ & 0.97 & 0.81 & 7.83 & 0.86 & 1.21 & 0.18 & 0.88 & 0.04 \\
\hline $02166-5026$ & 10611 & G5V & 16.9 & 14.7 & 1.00 & 0.82 & 9.04 & 0.75 & 1.22 & 0.74 & 1.19 & 0.52 \\
\hline $02390-5811$ & 12351 & MOVe & 59.4 & $57.0^{*}$ & 0.60 & 0.55 & 9.48 & 1.52 & & $\ldots$ & 0.45 & 0.03 \\
\hline $04007+2023$ & 18719 & G4V & 16.1 & $14.7^{*}$ & 1.12 & 0.53 & 8.66 & 0.75 & 5.17 & & 2.36 & 0.73 \\
\hline $04049-3527$ & $19052 \mathrm{~B}$ & & 14.0 & 16.4 & 0.97 & 0.71 & 9.14 & 0.70 & 2.06 & 0.41 & 1.47 & 0.09 \\
\hline $04074-6413$ & 19233 & G3V & 42.3 & 40.3 & 1.14 & 0.53 & 6.37 & 0.70 & & & 3.79 & 0.68 \\
\hline $04107-0452$ & 19508 & G3/5IV & 16.1 & $16.8^{*}$ & 1.24 & 1.08 & 7.37 & 0.71 & 0.80 & 0.07 & 0.55 & 0.31 \\
\hline $04422+0259$ & 21880 & Á0 & 5.7 & $6.8^{*}$ & 1.83 & 1.58 & 7.26 & 0.21 & 0.74 & 0.56 & 0.28 & 0.16 \\
\hline $05086-1810$ & 23932 & M5 & 107.8 & $118.5^{*}$ & 0.34 & 0.29 & 10.28 & 2.64 & 0.40 & 0.56 & 0.24 & 0.15 \\
\hline 05103-0736 & 24076 & F8 & 17.4 & $18.6^{*}$ & 1.15 & 1.08 & 7.42 & 0.62 & 0.35 & 0.14 & 0.20 & 0.02 \\
\hline $05525-0217$ & 27758 & F5 & 20.1 & $17.6^{*}$ & 1.29 & 0.97 & 7.26 & 0.63 & 1.62 & 0.28 & 1.33 & 0.08 \\
\hline $06454-3148$ & $32366 \mathrm{~B}$ & M3? & 39.6 & & 0.25 & 0.15 & 13.60 & 2.70 & & & 1.17 & 1.25 \\
\hline 06533-1902 & 33077 & A9/F0III & 10.5 & $11.7^{*}$ & 2.17 & 1.22 & 5.65 & 0.32 & 2.95 & 0.20 & $\ldots$ & $\ldots$ \\
\hline $07269+2015$ & 36156 & F2Vn & 7.3 & $7.6^{*}$ & 2.17 & 2.13 & 5.94 & 0.39 & 0.09 & 0.15 & & $\ldots$ \\
\hline $08122+1739$ & 40167 & G0V & 39.9 & 40.8 & 1.54: & $0.75:$ & 4.67 & 0.60 & 4.33 & & 2.66 & $\ldots$ \\
\hline $08403+1921$ & 42542 & A9V & 7.2 & 8.8 & 1.91 & 1.35 & 6.76 & 0.32 & 1.78 & 0.17 & & $\ldots$ \\
\hline $08447-4238$ & 42916 & G5V & 39.6 & 37.5 & 0.99 & 0.74 & 7.20 & 0.81 & 1.88 & 0.47 & 1.14 & \\
\hline $09252-1258$ & 46191 & $\mathrm{~K} 1 / 2 \mathrm{~V}$ & 23.0 & $22.9 *$ & 0.78 & 0.66 & 9.62 & 1.00 & 1.17 & 0.39 & 0.38 & 0.20 \\
\hline $10121-0241$ & 49969 & M0 & 81.1 & 86.3 & 0.44 & 0.25 & 10.64 & 2.32 & & & 1.00 & 0.05 \\
\hline $10294+1211$ & 51360 & F2 & 11.0 & $11.3^{*}$ & 1.53 & 1.18 & 7.25 & 0.51 & 1.43 & 0.08 & 1.21 & 0.15 \\
\hline $10529-1717$ & 53206 & G6V & 25.5 & $24.0^{*}$ & 1.10 & 1.03 & 7.13 & 0.74 & 0.36 & 0.17 & 0.25 & \\
\hline $11420-1701$ & 57078 & F5V & 17.0 & 17.2 & 1.27 & 0.67 & 7.61 & 0.56 & 3.98 & 0.54 & 2.86 & 0.31 \\
\hline $12485-1543$ & 62505 & $\mathrm{~K} 2 \mathrm{~V}$ & 42.0 & $37.8^{*}$ & 0.87 & 0.70 & 7.93 & 0.99 & 1.43 & 0.15 & 1.07 & 0.13 \\
\hline $13137-6248$ & 64537 & G5 & 15.7 & 15.8 & 0.94 & 0.83 & 9.14 & 0.83 & 0.76 & 0.21 & 0.31 & 0.18 \\
\hline $14330-4224$ & 71140 & A7/8IV & 7.8 & 9.0 & 1.45 & 1.31 & 7.78 & 0.32 & 0.56 & 0.32 & 0.32 & 0.33 \\
\hline $15245-1322$ & 75416 & K7V & 24.1 & 20.9 & 0.71 & 0.67 & 10.27 & 1.26 & & & 0.31 & 0.16 \\
\hline $15339-1700$ & 76203 & G9IV-V & 25.9 & 28.0 & 0.94 & 0.72 & 8.14 & 0.88 & 1.78 & 0.06 & 1.36 & $\ldots$ \\
\hline $17176+1025$ & 84595 & G5 & 14.4 & $17.7^{*}$ & 1.04 & 0.81 & 8.47 & 0.86 & & & 1.10 & $\ldots$ \\
\hline $17195-5004$ & 84759 & F2III & 16.9 & $14.2^{*}$ & 1.56 & 1.50 & 6.27 & 0.48 & 0.23 & 0.22 & $\ldots$ & $\ldots$ \\
\hline $17447-4244$ & 86847 & $\mathrm{~A} 2 / \mathrm{A} 3 \mathrm{IV} / \mathrm{V}$ & 13.9 & 12.9 & 1.89 & 1.48 & 5.87 & 0.18 & 1.24 & 0.08 & & \\
\hline $18040+0150$ & 88481 & G5 & 29.0 & 29.0 & 0.92 & 0.74 & 8.14 & 0.83 & 1.37 & 0.14 & 1.00 & 0.07 \\
\hline $18520-5418$ & 92592 & G3V & 19.5 & 21.5 & 0.98 & 0.65 & 8.54 & 0.69 & 2.81 & 0.18 & 1.75 & 0.43 \\
\hline $19377-4128$ & 96545 & F3IV & 6.8 & $7.9^{*}$ & 1.56 & 1.43 & 7.65 & 0.51 & 0.46 & 0.05 & 0.25 & 0.10 \\
\hline $19598-0957$ & 98416 & F5V & 45.0 & $41.2^{*}$ & 1.18 & 0.91 & 5.88 & 0.67 & 1.57 & 0.04 & 1.46 & \\
\hline $20212-5147$ & 100356 & K7 & 41.7 & $40.4^{*}$ & 0.59 & 0.56 & 10.23 & 1.73 & & & 0.34 & 0.18 \\
\hline $21099-2424$ & 104476 & G5V & 19.1 & $19.5^{*}$ & 1.03 & 0.92 & 8.09 & 0.79 & 0.68 & 0.28 & 0.32 & 0.15 \\
\hline $21368-3043$ & 106701 & G5V & 16.1 & $19.0^{*}$ & 0.94 & 0.86 & 8.67 & 0.76 & 0.58 & 0.62 & 0.34 & 0.42 \\
\hline $21400-5222$ & 106978 & F7III & 5.8 & $6.0^{*}$ & 2.34 & 2.20 & 6.20 & 0.67 & 0.27 & 0.19 & 0.32 & \\
\hline $21504-5818$ & 107806 & G6V & 24.1 & $21.8^{*}$ & 1.00 & 0.94 & 7.89 & 0.78 & 0.39 & 0.17 & 0.20 & 0.13 \\
\hline $22228-2937$ & 110483 & G6V & 25.5 & $25.5^{*}$ & 0.98 & 0.67 & 8.19 & 0.80 & 2.50 & 0.16 & 1.92 & 0.16 \\
\hline $22535-1137$ & 113031 & $\mathrm{~B} 8 \mathrm{IV} / \mathrm{V}$ & 4.0 & $6.0^{*}$ & 2.62 & 2.29 & 5.80 & $\ldots$ & 0.62 & 0.13 & 0.58 & $\ldots$ \\
\hline
\end{tabular}

